\title{
FORMULATION AND EVALUATION OF NANOSTRUCTURED LIPID CARRIERS BASED ANTI-INFLAMMATORY GEL FOR TOPICAL DRUG DELIVERY SYSTEM
}

\author{
ROHINI S KHARWADE ${ }^{1 *}$, NILESH M MAHAJAN ${ }^{2}$ \\ ${ }^{1}$ Department of Pharmaceutics, Dadasaheb Balpande College of Pharmacy, Besa, Nagpur, Maharashtra India. ${ }^{2}$ Department of \\ Pharmaceutical Sciences, Rashtrasant Tukadoji Maharaj Nagpur University, Nagpur, Maharashtra India. Email: rohinismore@gmail.com
}

Received: 11 January 2019, Revised and Accepted: 18 March 2019

\section{ABSTRACT}

Objective: Nanostructured lipid carrier (NLC)-based topical gel of lornoxicam (LXM) was formulated with the aim of controlled release action and to reduce systemic side effect for the treatment of an arthritic condition.

Methods: NLCs developed using high-pressure homogenization method and optimized using a $3^{2}$ factorial design with response surface methodology using design expert software. NLCs were characterized for particle size, zeta potential analysis, drug entrapment efficiency, and in vitro drug release studies to select the optimized formulation. The NLCs were suitably gelled and evaluated with respect to homogeneity, pH, viscosity, gel strength, spreadability, rheological characteristics, drug content, in vitro diffusion, and stability study. Safety of the NLC-based gel was assessed using primary skin irritation studies, and efficacy was confirmed using carrageenan-induced rat paw edema model.

Results: NLCs formulation comprising $2 \%$ of lipid (60:40) and surfactant (1.50\%) was confirmed as an optimized batch having a particle size $(138.2 \pm 3.60 \mathrm{~nm})$ with polydispersibility index value $0.344 \pm 0.034$. The zeta potential value indicates good physical stability. Based on the results from the in vitro release study it was shown that the formed gels had the ability to extend release of LXM for $24 \mathrm{~h}$ and showing percentage drug release of $90.92 \% \pm 1.96 \%$ at the end of $24 \mathrm{~h}$. Skin irritation studies revealed that the optimized gel formulation shows no erythema, edema, or ulceration.

Conclusion: The overall results of the present study clearly indicated promising potentials of NLC-based gel for delivering LXM topically over the conventional gel.

Keywords: Nanolipid carrier, Lornoxicam, Response surface methodology, Topical gel.

(c) 2019 The Authors. Published by Innovare Academic Sciences Pvt Ltd. This is an open access article under the CC BY license (http://creativecommons. org/licenses/by/4. 0/) DOI: http://dx.doi.org/10.22159/ajpcr.2019.v12i4.32000

\section{INTRODUCTION}

In the past decade, solid lipid nanoparticles (SLN) and nanostructured lipid carriers (NLC) have been looked on as promising carriers for presenting several attractive features for transdermal drug delivery [1]. These carriers are composed of physiological and biodegradable lipids exhibiting low systemic toxicity and low cytotoxicity. The small size of the lipid particles ensures close contact with stratum corneum and can increase the amount of drug penetrating into mucosa or skin. Due to their solid lipid matrix, a controlled release from these carriers is possible [1,2]. This becomes an important tool when it is necessary to supply the drug over a prolonged period of time, to reduce systemic absorption, and when drug produces irritation in high concentrations. To overcome some of the limitations of SLNs, namely limited drug loading and drug leakage during storage, NLCs have been developed $[3,4]$. They consist of a solid lipid matrix with a high content of liquid lipid. NLCs were studied and introduced with an intention to overcome the limitations of SLNs such as limited drug loading and drug leakage during storage. NLCs are the new generation of lipid nanoparticles, attracting major attention as novel colloidal drug carriers for topical use $[4,5]$.

Lornoxicam (LXM) is a nonsteroidal anti-inflammatory drug (NSAID) belonging to an oxicam class having potent analgesic and antiinflammatory effects. The mechanism of action of LXM, like that of other NSAIDs, is mainly due to the inhibition of prostaglandin biosynthesis through the inhibition of the cyclooxygenase (COX) enzymes COX-l and COX-2 [6]. It is recommended widely for symptomatic treatment of pain and inflammation linked with rheumatoid arthritis, osteoarthritis and proven to have efficacy in the management of pre-operative and postoperative pain associated with abdominal, bone-related, gynecological, and dental surgeries $[7,8]$. It requires repeated oral administration is due to to its short duration of action and low elimination half-life. The major well-known side effects of all NSAIDs are gastric irritation and peptic ulcers [8].

Hence, in the present investigation, the feasibility of NLC as a novel carrier system for topical application of LXM, with regard to the prolong of the release and bypass the hepatic first-pass effect was checked. Thus, the possibility of delivering LXM through the skin for local inflammation at low doses is beneficial. The role played by the oily phase component of the NLC was also judged by comparing the in vitro release. To increase the therapeutic efficacy of topically applied drugs, LXM-NLCs were incorporated within the gel matrix which was expected to deliver the drug for the extended period of time.

\section{MATERIALS AND METHODS}

Materials

LXM was obtained as a giftsample from Alkem Laboratories Ltd., Mumbai. Precirol ATO 5 was gifted from Gattefosse India Pvt., Ltd., Pluronic F-68 was gift sample by BASF ChemTrade GmbH (Burgbernheim, Germany)., Oleic acid by Loba Chemie, Mumbai, Carbopol-940 was procured from HiMedia Lab, Mumbai. Other required chemicals were of analytical grade procured from S.D Fine Chemicals, Mumbai, India.

\section{Animals}

Albino Wistar rats were used after obtaining the due permission for conducting in vivo studies, from Institutional Ethics Committee (Dadasaheb Balpande College of Pharmacy, Nagpur, India). The protocol according to form B was approved by Committee for the Purpose of Control and Supervision of Experiments on Animals (CPCSEA) and 
Institutional Animal Ethics Committee (Registration number 1426/PO/ $\mathrm{Re} / \mathrm{S} / 11 / \mathrm{CPCSEA})$.

\section{Screening of lipid}

Before the formulation of NLC's, screening of components should be performed to determine the most suitable solid lipid and liquid lipid that could be used in the preparation of NLCs, to give high entrapment of the drug and maximum drug loading into the lipid matrix. For this purpose, the solubility of LXM was determined in different solid lipids, oils, and surfactants. The solubility in solid lipids, i.e., stearic acid, compritol 888, and Precirol AT05(1000 mg) was taken in each test tube, the weighed amount of drug was added into the test tubes which were heated in a controlled temperature water bath kept at $80^{\circ} \mathrm{C}$. The drug was added until the saturation was achieved. The remaining amount of the drug was weighed again, and by that way, the quantity required to dissolve drug was found out [7-9].

\section{Design for optimizing formulations}

To obtain the optimal formulations with appropriate drug encapsulation efficiency and mean particle size of LXM-NLC, a $3^{2}$ factorial design based on a response surface methodology (RSM) was used to evaluate the effects of lipid concentration (X1) and surfactant concentration (X2). Objectives of this study were to establish the functional relationships between two operating variables of solid lipid concentration (X1) and surfactant concentration (X2) and two responses of encapsulation efficiency and mean particle size of NLC, respectively. To optimize LXM-NLC, mathematical model equations were derived by computer simulation programming Design-Expert ${ }^{\circledR}$ 8.0.1. For a better understanding of the two variables for the optimal LXMNLC performance, the models were presented as three-dimensional (3D) response surface graphs $[7,8]$. To carry out the above-mentioned study, for each factor (operating variable), the experimental range was selected on the basis of the results of preliminary experiments and the feasibility of preparing the NLC at the extreme values as shown in Table 1.

\section{Drug-excipients interaction study}

The drug-excipients interaction study was carried out using Fouriertransform infrared (FT-IR) and differential scanning calorimetry (DSC). FTIR spectra of LXM, Precirol ATO-5 (PRE) and physical mixture of lipids with LXM were studied using FTIR spectrophotometer (Shimadzu 8400S, Japan) at 10-12 tones pressure and thermal analysis was carried out using DSC (Mettler DSC 1 star system, Mettler-Toledo, Switzerland) at a heating rate of $10^{\circ} \mathrm{C} / \mathrm{min}$. The measurements were performed at a heating range of $40-300^{\circ} \mathrm{C}$ under nitrogen atmospheres [9].

\section{Method of preparation of NLC's}

The NLC was prepared by a modified method of hot high-pressure homogenization technique. LXM was dispersed in the lipid phase (consisted of Precirol ATO-5 and Oleic acid in 60:40 ratio) and kept in a heating water bath at $80^{\circ} \mathrm{C}$ [10]. The aqueous phase comprised surfactant (poloxamer188) dissolved in water. Both the phases were maintained at a temperature above the melting point of the lipid $\left(80^{\circ} \mathrm{C}\right)$. At this temperature, the melted hot lipid phase was then dispersed in the hot surfactant phase obtaining a pre-emulsion under mechanical

Table 1: Predicted optimum ranges for independent factors

\begin{tabular}{lll}
\hline $\begin{array}{l}\text { Formulation } \\
\text { codes }\end{array}$ & $\begin{array}{l}\text { \% Lipid } \\
\text { concentration (X1) }\end{array}$ & $\begin{array}{l}\text { \% Surfactant } \\
\text { concentration (X2) }\end{array}$ \\
\hline F1 & 3.00 & 1.00 \\
F2 & 1.00 & 1.50 \\
F3 & 2.00 & 1.00 \\
F4 & 2.00 & 1.50 \\
F5 & 3.00 & 1.50 \\
F6 & 2.00 & 0.50 \\
F7 & 1.00 & 0.50 \\
F8 & 1.00 & 1.00 \\
F9 & 3.00 & 0.50 \\
\hline
\end{tabular}

stirring (Remi Instruments Ltd., Mumbai, India) at $800 \mathrm{rpm}$ at $80^{\circ} \mathrm{C}$ for $30 \mathrm{~min}$.

The warm pre-emulsion was introduced into a high-pressure homogenizer (PANDA 2K, NiroSoavi, Italy) at 700 bar pressure and three cycles to form the NLC dispersion. Then, the formulated NLC was allowed to cool at room temperature which was further used for characterization $[9,10]$.

\section{Preparation of LXM loaded NLC gel}

LXM loaded NLC gel was prepared using different gelling agents such as Carbopol-940 and xanthan gum. Based on the compatibility with nanoparticulate dispersion, feel esthetic appeal and ease of spreadability Carbopol 940 were selected as the gelling agent. Different concentrations of Carbopol 940 ranging from 0.50 to $1 \%$ were used for gelling, and the concentration giving the optimum viscosity $(0.7 \%)$ was chosen for the formulation of gel. Carbopol 940 was added to the nanoparticle dispersion under mechanical stirring at $800 \mathrm{rpm}$. Stirring was continued until the Carbopol 940 got dispersed. The Carbopol dispersion was neutralized using $0.2 \mathrm{~mL}$ triethanolamine [11].

\section{Evaluation and characterization}

Particle size analysis was performed by photon correlation spectroscopy with a Malvern Zetasizer (Nano ZS 90, Malvern Ltd., UK). The NLCs were evaluated for the rheological behavior using Brookfield Viscometer (RVDV Pro II). Entrapment efficiency (EE) and drug loading could be achieved by the following equations $[12,13]$.

$$
\begin{aligned}
& \mathrm{EE} \%=\frac{\text { Total Drug }- \text { Free Drug }}{\text { Total Drug }} \times 100 \\
& \mathrm{DL} \%=\frac{\text { Initial Drug }- \text { Free Drug }}{\text { MixedLipid }} \times 100
\end{aligned}
$$

\section{In vitro drug diffusion study}

In vitro drug diffusion study was performed using the diffusion cell assembly. The drug-loaded NLC gel was evaluated using dialysis membrane (molecular weight cutoff $>12,000$, HiMedia) as a barrier containing $\mathrm{pH} 7.4$ phosphate buffer solution as a media at $371 \mathrm{~nm}$ wavelength [10-13]

\section{Ex vivo skin irritation studies}

The albino Wistar rats were divided into two groups with three rats in each group. On the previous day of the experiment, the hairs on the back side area of rats were removed. The animals of Group I were served as control, without any treatment. Other group of animals (Group II) was applied with prepared NLCs based gel. Gel was applied once a day for 7 days, and the site was covered with a cotton bandage and observed for any sensitivity. Score of erythema is read and recorded as Score 0 for no erythema; Score 1 for mild erythema (barely perceptible- light pink); Score 2 for moderate erythema (dark pink); and Score3 for severe erythema (extreme redness) [14]

\section{In vivo studies}

In vivo study of the anti-inflammatory drug was conducted on adult male Wistar rats having a weight range between 130 and $150 \mathrm{~g}$. The animals were divided into three groups, each group containing three animals. Group I received a topical saline application (control group), Group II received marketed diclofenac gel, and Group III received LXM-NLC gel. The volume of paw edema ( $\mathrm{ml}$ ) was measured for each animal using a digital plethysmometer 2.0 (V.J. Instrument, Nashik, India). The rats were marked on the left hind paw just beyond the tibia-tarsal junction, and every time the paw was dipped in the electrolyte fluid column up to a fixed mark to make constant paw volume. The tested preparations were applied to the left hind paws of rats for $30 \mathrm{~min}$ before the initiation of inflammation by carrageenan. The initial paw volume of the rats was measured just before carrageenan injection, and the increase in volume due to fluid displacement was noted from a digital display, followed by an injection of $0.1 \mathrm{ml}$ of $1 \%(\mathrm{w} / \mathrm{v})$ carrageenan solution in saline 
in subplantar region of left hind paws of the rats. Measurement of paw volumes was done after $1,2,3,4$, and $5 \mathrm{~h}[15,16]$. The percent inhibition rate was calculated as follows:

$$
\% \text { Inhinition of edema }=\frac{\mathrm{V} \text { control }-\mathrm{V} \text { treated }}{\mathrm{V} \text { control }} \times 100
$$

\section{Stability study}

Stability testing of the drug product is a part of drug discovery and ends with the commercial product, to assess the drug and formulation stability, stability studies were done. The stability study was carried out for the most satisfactory formulation. The optimized gel formulation was sealed in a glass vial and kept at $30+2^{\circ} \mathrm{C}$ at $65+5 \% \mathrm{RH}$ for 1 months and analyzed. At the 1 month ending, the samples were analyzed for the drug content, $\mathrm{pH}$ determination, viscosity, and in vitro diffusion study.

\section{RESULTS AND DISCUSSION}

\section{Selection of lipids}

Based on the screening of lipids, for the maximum solubility of the drug in Precirol was selected as solid lipid for the formulation of NLC's and has property such as good flow property, non toxicity, approved regulatory status, and cost which also favors its choice as solid lipid, oleic acid was screened out as most suitable liquid lipid to be used in the LXM-NLCs and poloxamer 188 was selected as a surfactant phase for the formulation of NLCs.

\section{Drug-excipients interaction study}

The possible interaction between the drug and the ingredients used in the preparation of the NLC was studied by FTIR and DSC. All the characteristic peaks of the drug appeared in the spectra of the physical mixture at the same wavenumber indicating no modification or interaction between the drug and the polymer shown in Fig. 1.

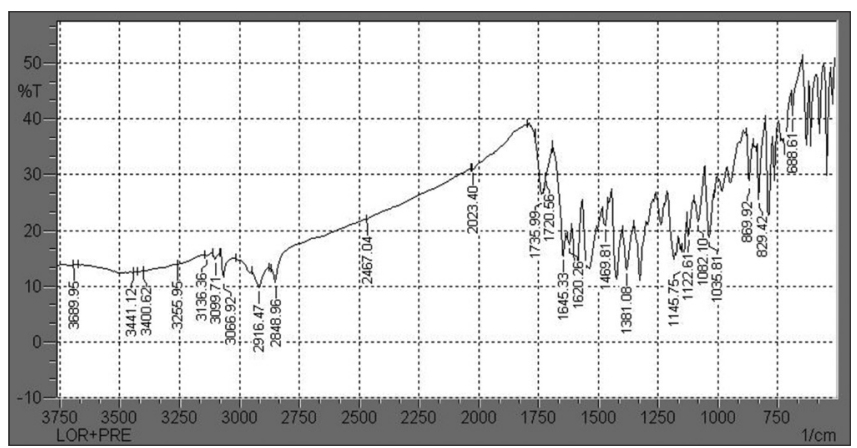

Fig. 1: Infrared spectrum of the physical mixture of lornoxicam and Precirol
The DSC heating and cooling curves were recorded as a plot of enthalpy (in $\mathrm{mW}$ ) versus temperature. For the LXM, the melting process took place at $218.75^{\circ} \mathrm{C}$ with a maximum peak at $220.66^{\circ} \mathrm{C}$ and for bulk material of Precirol melting process took place at $52.93^{\circ} \mathrm{C}$ with a maximum peak at $59.93^{\circ} \mathrm{C}$. No significant change in the position of peaks was observed after running the physical mixture (1:1) of drug and solid lipid. Thus, the physical incompatibility between the components was discarded.

In the DSC thermogram of lyophilized LXM-NLCs, a small endothermic peak was observed around at $58^{\circ} \mathrm{C}$ due to the lipid Precirol as seen in Fig. 2. The disappearance of the exothermic peak of LXM in the LXMNLCs powder suggests that the drug is completely encapsulated in the lipid matrix and converted to amorphous state from crystalline state.

\section{Preparation of NLCs}

LXM-NLCs were prepared using hot high-pressure homogenizer as it is the best method used in the scale up production (Muller et al., 2000). Indeed, it has been reported that lipid nanoparticles stabilized with surfactant mixtures have lower particle sizes and higher storage stability compared to formulations with only one surfactant (Mehnert and Maeder, 2001). It was observed from the preliminary studies that 500 bars of pressure are enough to produce nanoparticles having a particle size of $200-300 \mathrm{~nm}$. While the number of cycles through high-pressure homogenization was fixed to four cycles, it was seen that by increasing the number of cycles there was an increase in the surface kinetic energy over the particles leading to aggregation of the nanoparticle causing an increase in particle size $[13,14]$.

\section{Optimization and validation of formulas for the preparation LXM- NLCs}

The compositions of different batches of LXM-NLC by $3^{2}$ factorial design are summarized in Table 1 . Table 2 showed the experimental results concerning the tested variables on drug encapsulation efficiency (EE) and mean diameter of particle size. The two dependent values ranged from $98.38 \%$ to $99.51 \%$ by weight and 138.2 to $-236.3 \mathrm{~nm}$. A mathematical relationship between factors and parameter was generated by response surface regression analysis using DesignExpert $^{\circledR}$ 8.0.1 software for all nine formulation. The ranges of response $\mathrm{Y} 1$ and Y2 were $138.2-236.3 \mathrm{~nm}$ and 98.38-99.51\%, respectively.

Regression equations for fitted quadratic interactive model $\mathrm{Y} 1=148.37+18.50 \mathrm{X} 1-16.92 \mathrm{X} 2+3.05 \mathrm{X} 1 \mathrm{X} 2+44.10 \mathrm{X} 12+12.65 \mathrm{X} 22$

$$
\mathrm{Y} 2=94.61+3.31 \mathrm{X} 1+0.37 \mathrm{X} 2
$$

It was observed that the best fitted models were quadratic for both particle size (Y1) and EE (Y2). Quadratic model is selected because it shows the interactive effect of all the independent variables on

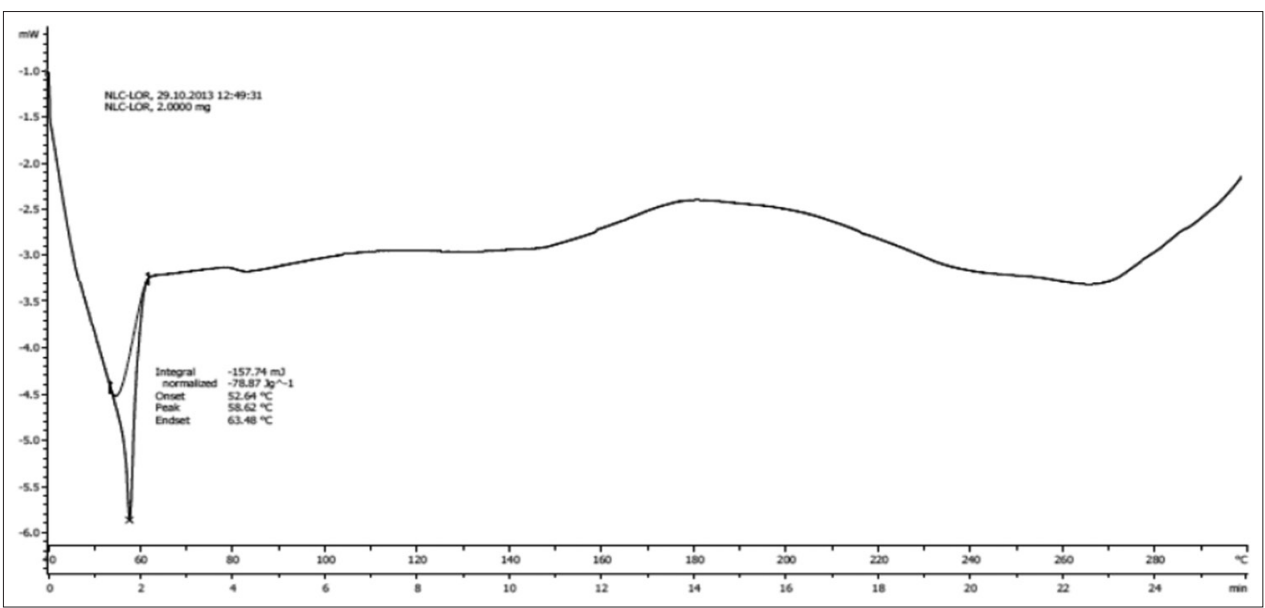

Fig. 2: Differential scanning calorimetry thermogram of freeze-dried nanostructured lipid carrier formulation of lornoxicam 


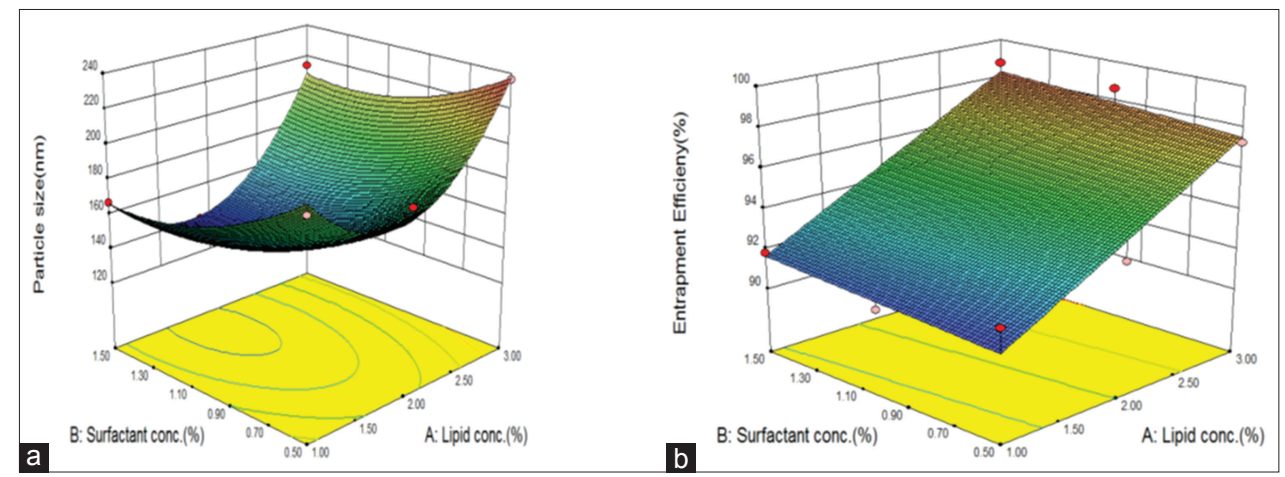

Fig. 3: Response surface plot showing the effect of lipid concentration with respect to surfactant concentration over mean particle size (a) and entrapment efficiency (b)

Table 2: Summary of results of regression analysis for responses Y1 and Y2

\begin{tabular}{llllll}
\hline Models & $\mathbf{R}^{2}$ value & Adjusted $\mathbf{R}^{2}$ & Predicted $\mathbf{R}^{2}$ & S.D & CV\% \\
\hline Response (Y1) quadratic & 0.9780 & 0.9414 & 0.7331 & 7.75 & 4.16 \\
Response (Y2) quadratic & 0.9436 & 0.9249 & 0.8601 & 0.81 & 0.86 \\
\hline
\end{tabular}

Table 3: Particle size, PDI, and entrapment efficiency of formulated NLCs

\begin{tabular}{llll}
\hline Batch code & Particle size $(\mathbf{n m})$ & Polydispersity index (PDI) & Entrapment efficiency (\%) \\
\hline F1 & $207.0 \pm 2.51$ & $0.298 \pm 0.054$ & $98.64 \pm 1.45$ \\
F2 & $167.4 \pm 1.52$ & $0.359 \pm 0.046$ & $91.83 \pm 2.13$ \\
F3 & $147.5 \pm 3.05$ & $0.399 \pm 0.032$ & $94.45 \pm 2.54$ \\
F4 & $138.2 \pm 3.60$ & $0.344 \pm 0.034$ & $96.90 \pm 1.98$ \\
F5 & $214.9 \pm 2.10$ & $0.471 \pm 0.026$ & $99.21 \pm 1.76$ \\
F6 & $184.7 \pm 2.60$ & $0.437 \pm 0.045$ & $93.22 \pm 2.17$ \\
F7 & $201.0 \pm 2.64$ & $0.485 \pm 0.047$ & $92.11 \pm 3.11$ \\
F8 & $178.8 \pm 2.08$ & $0.381 \pm 0.052$ & $90.80 \pm 3.01$ \\
F9 & $236.3 \pm 3.04$ & $0.288 \pm 0.044$ & $97.31 \pm 1.65$ \\
\hline
\end{tabular}

PDI: Polydispersibility index, NLC: Nanostructured lipid carrier

responses that linear/non-linear model does not show. The values of $\mathrm{R}^{2}$, adjusted $\mathrm{R}^{2}$, predicted $\mathrm{R}^{2}$, standard deviation, and percentage coefficient of variation are given in Table 2 along with the regression equation generated for each response. The results of ANOVA in the dependent variables demonstrate that $\mathrm{P}$ significance value for mean particle size and EE was 0.0108 and 0.002 , respectively, so that the model was significant for both the response variables.

\section{Response surface plots analysis}

The contour plot shows predicted particle size values at different levels of lipid concentration and surfactant concentration (Fig. 3a). It can be seen that the increase in lipid concentration increased mean particle size, whereas an increase in surfactant concentration decreased the mean particle size. The surface response curve also shows increased particle size with increasing amount of lipid concentration, whereas an increase in the amount of surfactant concentration, led to a little reduction in particle size. The contour plot shows predicted EE values at different levels of lipid concentration and surfactant concentration (Fig. 3b). It can be seen that increase in lipid concentration. Whereas increase in surfactant concentration. slightly increase in entrapment efficiency. The surface response curve also shows increased EE with an increasing amount of lipid concentration, whereas an increase in the amount of surfactant concentration, led to slightly increase in EE [15].

\section{Particle size analysis and polydispersibility index (PDI)}

The particle size of the NLCs suspension is a crucial factor because it determines the rate and extent of drug release as well as drug absorption. The smaller droplet size provides a larger interfacial surface area for drug absorption. In addition, the smaller droplet size permits a faster release rate. Furthermore, it has been reported that
Table 4: Data showing stability studies

\begin{tabular}{|c|c|c|c|}
\hline \multirow[t]{2}{*}{ Sr. No. } & \multirow[t]{2}{*}{ Parameters } & \multicolumn{2}{|c|}{ Storage period (1 month) } \\
\hline & & Initial & $\begin{array}{l}30+2^{\circ} \mathrm{C} \text { at } \\
65+5 \% \mathrm{RH}\end{array}$ \\
\hline 1 & Drug content (\%) & $94.99 \pm 1.35$ & $93.10 \pm 2.05$ \\
\hline 2 & $\mathrm{PH}$ & $7.2 \pm 0.20$ & $7.2 \pm 0.15$ \\
\hline 3 & Viscosity (cp) & $11654 \pm 108.55$ & $11265 \pm 110.05$ \\
\hline 4 & In vitro diffusion study (\%) & $90.92 \pm 1.96$ & $89.25 \pm 1.05$ \\
\hline
\end{tabular}

the smaller particle size of the emulsion droplets may lead to faster absorption and improve the bioavailability. Furthermore, PDI measures the width of particle size distribution. If PDI is lower than 0.1, it might be associated with high homogeneity in the particles, whereas high PDI values suggest a broad size distribution. The particle size and PDI of the designed batches are shown in Table 3 .

\section{Particle size analysis, PDI, and percent EE}

LXM- NLCs prepared in this work using the high-pressure homogenization technique showed high EE values with an increase in the percentage oleic acid content. The percentage EE of the resulting NLCs is presented in Table 4. It was observed that the increase in oil content, the percentage of encapsulated drug increases because the drug shows more solubility in the liquid lipid than the solid lipid as the number of imperfections in the crystal lattice increases in the presence of liquid lipid as compared to solid lipid. The high EE values observed in this study indicate that the lipid and surfactant compositions employed are very adequate for LXM entrapment in NLCs $[15,16]$. 
Table 5: Mean paw edema volume in rats

\begin{tabular}{|c|c|c|c|c|c|}
\hline \multirow{2}{*}{$\begin{array}{l}\text { Time } \\
\text { (hour) }\end{array}$} & \multirow{2}{*}{$\begin{array}{l}\text { Control } \\
\text { (saline) }\end{array}$} & Standard (diclofenac gel) & Test (LXM-NLC gel) & Standard (diclofenac gel) (\%) & Test (LXM-NLC gel) (\%) \\
\hline & & \multicolumn{2}{|l|}{ After carrageenan induced } & \multicolumn{2}{|l|}{ Percent inhibition edema rate } \\
\hline 1 & $1.37 \pm 0.15$ & $0.92 \pm 0.01$ & $0.87 \pm 0.03$ & $32.84 \pm 0.03$ & $36.49 \pm 0.02$ \\
\hline 2 & $1.57 \pm 0.03$ & $1.03 \pm 0.77$ & $0.95 \pm 0.01$ & $34.39 \pm 0.12$ & $39.49 \pm 0.01$ \\
\hline 3 & $1.82 \pm 0.01$ & $1.14 \pm 0.14$ & $1.09 \pm 0.02$ & $37.36 \pm 0.03$ & $40.10 \pm 0.05$ \\
\hline 4 & $1.89 \pm 0.12$ & $1.16 \pm 0.07$ & $1.05 \pm 0.14$ & $38.62 \pm 0.01$ & $44.44 \pm 0.10$ \\
\hline 5 & $1.94 \pm 0.02$ & $1.12 \pm 0.15$ & $1.02 \pm 0.02$ & $42.26 \pm 0.05$ & $47.42 \pm 0.15$ \\
\hline
\end{tabular}

All values represent mean \pm standard deviation $(n=3) \mathrm{P}<0.0001$ (compared with control). LXM: Lornoxicam, NLC: Nanostructured lipid carrier

\section{Zeta-potential measurement}

The zeta-potential of the optimized LXM-NLC was found to be -27.8 . It possesses negative surface charges due to the negatively charged Precirol. In addition to Precirol, surfactant (poloxamer 188) was also negatively charged. It is currently admitted that zeta potentials under $(-30) \mathrm{mV}$ are optimum and $<(-60) \mathrm{mV}$ are required for full electrostatic stabilization.

Optimized batch (F4) selected for the formulation of gel. The percent drug content of gel formulation was found to be $94.99 \pm 1.35 \%$. This showed that drug was uniformly distributed in the formulated gel with viscosity $11654 \pm 1.55 \mathrm{cp}, \mathrm{P}^{\mathrm{H}} 7.2 \pm 0.20$, spreadability $9.4 \pm 0.25$ (g.cm/s.), drug content $94.99 \pm 1.35 \%$, and gel strength $20 \pm 0.90 \mathrm{~s}$.

\section{Rheological measurements}

The apparent viscosity values were measured for gel formulations using Brookfield viscometer with spindle no. 64 at 10-100 rpm and 100-10 rpm. In the gel state, all the formulations were observed to be exhibiting the pseudoplastic flow. The characteristic concavity of the rheogram toward the shear rate axis indicates that developed formulation exhibited pseudoplastic flow. This pseudoplasticity results from a colloidal network structure that aligns itself in the direction of shear, thereby decreasing the viscosity as the shear rate increases showed in Fig. 4. NLC loaded gels showed that upcurve does not coincide with the down curve, meaning that the samples are non newtonian in nature showing thixotropic behavior, however, with lower structural breakdown [18].

\section{In vitro drug diffusion studies}

On the basis of percentage EE and particle size batch F4 were selected for in vitro drug diffusion study. The release profiles exhibited an inflection point, which indicated the gel formation in the donor compartment of the diffusion cell. The results showed that the formed gels had the ability to extend the release of LXM for the duration of about $24 \mathrm{~h}$. The cumulative percentage of drug diffused was observed for $24 \mathrm{~h}$, the sample was analyzed in triplicate. In Fig. 4 shows the cumulative percentage of drug diffusion patterns of the formulation. The amount of the polymer and the lipid may retard the drug diffusion giving more prolonged release of the drug [17, 18 ]. In $24 \mathrm{~h}$ of the drug diffusion study, the effect of the amount of polymer used and particle size of the formulation showed a direct relation with cumulative percentage drug diffused. The percentage drug diffused in first $8 \mathrm{~h}$ for the formulation was calculated, and the maximum diffusion was observed (53.08\%). The release was observed from LXM-NLC gel which showed sustained and steady release over the entire period of study.

The results obtained in in vitro release studies were plotted in different kinetic models. Regression coefficient $\left(\mathrm{R}^{2}\right)$ values for zeroorder 0.885, first-order 0.958, Higuchi Model 0.960, and KorsmeyerPeppas Model 0.970. This indicated that the release data were best fitted with Korsmeyer-Peppas kinetic plot model because the value of $\mathrm{R}^{2}$ is greater in this model $[18,19]$. The drug release data from the LXM-NLC's gel were fitted into Korsmeyer-Peppas model to find the release mechanism. Model fitting revealed that LXM-NLCs gel showed anomalous transport (non-Fickian) mechanism for the release of drug based on the " $n$ " value $(0.533$, which is $0.5<n<1)$, whereas $R^{2}$ was found to be 0.970 .

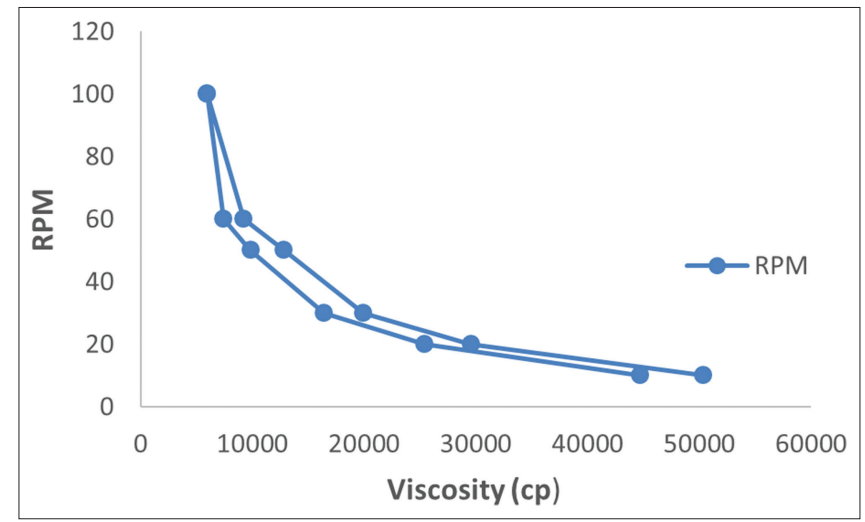

Fig. 4: Rheological behavior of gel formulation (F4)

\section{Skin irritation study}

Skin irritation studies carried out on albino Wistar rats revealed that the gel formulation shows no erythema, edema, or ulceration. It shows to be nonirritant [19]. Thus, this formulation was suitable for topical application resulted in selecting the gel for further tests.

\section{In vivo study (anti-inflammatory activities)}

The anti-inflammatory activity of the optimized formulation was evaluated by the carrageenan-induced hind paw inflammation method on Wistar rats. The percentage inhibition value of LXM-NLC gel was compared to diclofenac gel. The formulation gel not only decreased the inflammation by a larger magnitude but also sustained the effect for a prolonged period. Hence, the NLC-based gel formulation of LXM remained superior to the marketed product in its ability to suppress edema and sustained the anti-inflammatory activity [19].

The mean paw edema volume in rats before carrageenan induced was $0.75+0.05$ for control (Saline), $0.80 \pm 0.02$ for standard (diclofenac gel), and $0.82 \pm 0.02$ for test (LXM - NLC gel) and after carrageenan induced shown in Table 5

\section{Stability study}

In the stability study, after every 30 days, samples were withdrawn and retested for viscosity (cps) and drug content. The formulation did not show any significant change in both parameters. It indicates that this formulation was able to retain its stability for up to 3 months. Stability data are shown in Table 4.

\section{CONCLUSION}

NLC-based gel of LXM formulated in two-step process; first preparation of NLC dispersion followed by addition of the gelling agent. NLC dispersion was formulated by high-pressure homogenization method. As compared with the other methods, it showed the advantages of higher drug loading capacity, higher drug encapsulation efficiency, and no need for an organic solvent. Formulation comprising $2 \%$ of lipid (60:40) and surfactant (1.50\%) was confirmed as a optimized batch having minimum values for particle size $(138.2+3.60 \mathrm{~nm})$ with PDI value $0.344+0.034$. The zeta-potential was found to be -27.8 , indicating good physical stability. The optimized batch also shows good 
percentage EE of $96.90+1.98$. Topical NLCs based gel appeared to be an interesting approach to improve problems associated with oral delivery of LXM which having severe adverse effects.

The topical gel has suitable gel strength $(20+0.90 \mathrm{~s})$ so as to be administered easily and retained at skin without leakage after administration. Based on the results from the in vitro release study it was shown that the formed gels had the ability to extend the release of LXM for the duration of about $24 \mathrm{~h}$ and showing percentage drug release of $90.92+1.96$ at the end of $24 \mathrm{~h}$. Skin irritation studies carried out on albino Wistar rats revealed that the optimized gel formulation shows no erythema, edema, or ulceration. It shows to be nonirritant. Following application of gel in left hind paw in rats, formulation provided significant anti-inflammatory activity compared to a standard drug. The overall results of the present study clearly indicated promising potentials of NLC-based gel for delivering LXM topically over the conventional gel. From the stability study, it was observed that F4 batch is stable and used for topical application.

It can be finally concluded that formulating LXM-NLC gel considered a promising topical anti-inflammatory system and can be effectively used in acute inflammation and pain associated with arthritis.

\section{ACKNOWLEDGMENT}

The authors want to acknowledge the management of Dadasaheb Balpande College of Pharmacy, Nagpur University and R.C. Patel Institute of Pharmaceutical Education and Research, Shirpur, DistDhule, MS, India, for providing the research facility to carry out the research work.

\section{AUTHORS' CONTRIBUTIONS}

All the authors contributed equally in making the manuscript ready for submission.

\section{CONFLICTS OF INTEREST}

Authors declare no conflicts of interest.

\section{REFERENCES}

1. Brigger $C$, Dubernet $P$. Nanoparticles in cancer therapy and diagnosis. Adv Drug Deliv 2002;54:631-51.

2. Abather A, Sadiq A, Abdul R. Formulation and evaluation of silibinin loaded solid lipid nanoparticles for peroral use targeting lower part of gastrointestinal tract. Int J Pharm Pharm Sci 2014;6:55-67.

3. Ajialex R, Chacko J, Jose S, Souto B. Lopinavir loaded solid lipid nanoparticles (SLN) for intestinal lymphatic targeting. Eur J Pharm Sci
2011;42:11-8

4. Muller H. Solid-liquid (semi-solid) lipid particles and method of producing highly concentrated lipid particle dispersions. Eur Pat Appl 2000;45:199-203.

5. Muller R, Wissing A, Kayser O. Solid lipid nanoparticles for parenteral drug delivery. Adv Drug Deliv Rev 2004;56:1257-72.

6. Araujo J, Gonzalez E, Egea A, Garcia L, Souto B. Optimization and physicochemical characterization of a triamcinolone acetonide loaded NLC for ocular antiangiogenic applications. Int J Pharm Sci 2010; 393:167-75

7. Senthil P, Arivuchelvan A, Jagadeeswaran A, Subramanian N, Senthil C, Mekala P. Formulation, optimization and evaluation of enrofloxacin solid lipid nanoparticles for sustained oral delivery. Asian J Pharm Clin Res 2015;8:231-6.

8. Panchaxari M, Dandagi G, Dessai A, Gadad V. Formulation and evaluation of nanostructured lipid carrier (NLC) of lornoxicam. Int J Pharm Pharm Sci 2014;6:198-210.

9. Muchow M, Maincent P, Muller H. Lipid nanoparticles with a solid matrix $(S L N \circledast$, NLC $®$, LDC $®)$ for oral drug delivery. Drug Dev Ind Pharm 2008;34:1394-405.

10. Chalikwar S, Belgamwar V, Talele R, Surana S, Patil M. Formulation and evaluation of nimodipine-loaded solid lipid nanoparticles delivered via lymphatic transport system. Coll Sur Bioint 2012;97:109-6.

11. Singh H, Gupta RD, Gautam G. Formulation development, characterization, and in vitro-in vivo study of antihyperlipidemic drug rosuvastatin calcium-solid lipid nano particles. Asian J Pharm Clin Res 2018;11:436-43.

12. Nanjwade B, Kadam V, Manvi F. Formulation and characterization of nanostructured lipid carrier of ubiquinone (Coenzyme Q10). J Biom Nanotech 2013;9:450-60

13. Hu F, Jiang S, Du Y, Zeng S. Preparation and characterization of stearic acid nanostructured lipid carriers by solvent diffusion method in an aqueous system. Coll Sur Bioint 2005;45:167-73.

14. Zhang X, Liu J, Liu H, Ni J, Zhang W, Qiao H, et al. Formulation and optimization of dihydroartemisinin nanostructured lipid carries using response surface methodology. Powder Tech 2010;197:120-8.

15. Venkateswarlu V, Manjunath K. Preparation, characterization and in vitro release kinetics of clozapine solid lipid nanoparticles. J Contact Rel 2004;95:627-38.

16. Asmin B, Reddy P. Formulation and evaluation of dasatinib loaded solid lipid nanoparticles. Int J Pharm Pharm Sci 2018;10:14-20.

17. Kesharwani R, Sachan A, Singh S, Patel D. Formulation and evaluation of solid lipid nanoparticle based topical gel of etoricoxib. J App Pharm Sci 2016;6:124-31.

18. Montenegro L, Campisi A, Sarpietro M, Carbone C, Acquaviva R, Raciti $\mathrm{G}$, et al. In vitro evaluation of idebenone-loaded solid lipid nanoparticles for drug delivery to the brain. Drug Dev Ind Pharm 2011; 37:737-47.

19. Singh S, Singh S, Sharma A, Kaur D, Katual M. Formulation and in-vitro evaluation of solid lipid nanoparticles containing levosulpiride. Open Nanomed J 2017;4:17-29. 